\title{
Les migrants des politiques climatiques : nouveaux défis face aux déplacements générés par le changement climatique
}

The Climate Policy Migrant: New Challenges in Climate Change Related

Displacement

Jeanette Schade

\section{(2) OpenEdition \\ Journals}

\section{Édition électronique}

URL : http://journals.openedition.org/conflits/18584

DOI : $10.4000 /$ conflits. 18584

ISSN : $1777-5345$

Éditeur :

CCLS - Centre d'études sur les conflits lilberté et sécurité, L'Harmattan

Édition imprimée

Date de publication : 30 décembre 2012

Pagination : 85-110

ISBN : 978-2-343-00589-8

ISSN : 1157-996X

Référence électronique

Jeanette Schade, «Les migrants des politiques climatiques : nouveaux défis face aux déplacements générés par le changement climatique », Cultures \& Conflits [En ligne], 88 | hiver 2012, mis en ligne le 15 mars 2014, consulté le 30 mars 2021. URL : http://journals.openedition.org/conflits/18584; DOI : https://doi.org/10.4000/conflits.18584 


\section{Les migrants des politiques climatiques : nouveaux défis face aux déplacements générés par le changement climatique 1}

\section{Jeanette SCHADE}

Dr. Jeanette Schade est chercheur au Center on Migration, Citizenship and Development (COMCAD) de l'Université de Bielefeld. Elle y enseigne les questions environnementales, les migrations et les conflits. Depuis 2011, elle est la viceprésidente de l'action COST sur le changement climatique et les migrations, financée par la European Cooperation in Science and Technology.

Walgré les efforts des chercheurs spécialistes des migrations, les 1 «migrants climatiques » sont encore couramment perçus comme des gens déplacés en raison des impacts négatifs du changement climatique. Ce récit sur la cause «climatique » comme principal facteur de migration découle en grande partie de l'influence qu'exercent sur le débat climatique les chercheurs spécialisés dans les études sur la population et l'environnement (P\&E). En effet, les approches néo-malthusiennes qui sous-tendent ce débat comportent le risque d'aboutir à des solutions non pertinentes comme cela s'est déjà produit dans les années 1970 avec les politiques suivies en matière de sécurité alimentaire. Une des solutions discutée aujourd'hui est celle du déplacement planifié des populations (réinstallation/relocalisation). Comme les questions de la «migration climatique » et du déplacement planifié ont trouvé une certaine reconnaissance au cours des négociations de la $16^{\mathrm{e}}$ conférence des parties 2 (COP-16) au Mexique en 2010, il y a de fortes chances pour que des politiques soient développées en ce sens au sein de la Convention-cadre des Nations Unies sur les changements climatiques (CCNUCC). Malheureusement, ces politiques risquent de se focaliser sur la relocalisation, qui est un outil à double tranchant mobilisé dans de nombreux programmes

1. Texte traduit de l'anglais par Angélique Petrits. Toutes les traductions des citations anglaises sont du même traducteur.

2. La conférence des parties et l'organe suprême de la Convention-cadre des Nations Unies sur les changements climatiques (CCNUCC). Composée de tous les États parties de la Convention, elle veille à la bonne application des ses objectifs. 
portant sur l'atténuation et l'adaptation au changement climatique. L'expérience montre que cette solution échoue bien souvent et que ses bénéficiaires supposés se retrouvent parfois dans des situations plus difficiles qu'auparavant. Le présent article suggère qu'une approche des déplacements planifiés dans le cadre de la CCNUCC fondée sur les droits de l'Homme pourrait renforcer la protection des personnes vulnérables confrontées à des projets de réinstallation.

L'hypothèse soutenue ici est explicitée en cinq étapes. Nous nous intéresserons tout d'abord aux racines du discours sur la « rareté des ressources » qui caractérise les études sur la population et l'environnement. Nous montrerons ensuite que le débat sur la migration climatique et le déplacement planifié est dans une large mesure influencé par ce discours. Troisièmement, les défis et les pièges de la réinstallation seront illustrés à partir de différentes études de cas. Quatrièmement, nous revisiterons les travaux d'Amartya Sen qui contestent la pertinence de l'approche malthusienne dans le combat contre la famine dans le monde. Des leçons seront ainsi tirées à l'égard des personnes menacées de réinstallation et l'accent sera mis sur la nécessité d'établir un cadre protecteur des droits des groupes vulnérables afin de donner du poids à leur voix. Enfin, la place des droits de l'Homme et la CCNUCC seront brièvement présentées. L'élément le plus important qui ressort de cette analyse est que la réinstallation doit être évitée lorsque cela est possible.

L'article est basé dans une large mesure sur l'analyse du discours et adopte une approche critique. Les termes «migrations climatiques » et « migrant climatique » sont utilisés comme des outils pour mener l'analyse et non comme des termes ayant un contenu bien défini.

\section{L’influence des études Population \& Environnement (P\&E) sur les recherches en matière de changement climatique}

En raison de son origine anthropique, le débat sur le changement climatique est largement dominé par les sciences naturelles et par les études sur la population et l'environnement. L'attention est clairement portée sur le lien entre consommation des ressources fossiles et émissions de gaz à effet de serre (GES), et ses conséquences sur l'atmosphère (impacts de premier ordre), le climat (impact de second ordre), "les systèmes naturels » (impact de troisième ordre) et les "systèmes humains » (impact de quatrième ordre). En ce qui concerne les mesures à adopter, les premiers rapports d'évaluation du Groupe d'experts intergouvernemental sur l'évolution du climat (GIEC) dans les années 1990 ont souligné le besoin de limiter les GES (mesures d'atténuation). L'adaptation est essentiellement limitée à la gestion des ressources et à des solutions techniques telles que la productivité agricole, l'aménagement, la construction de barrages, etc. ${ }^{3}$. 
La place accordée à l'adaptation dans le débat depuis le début de ce millénaire s'explique par un intérêt croissant pour la question de vulnérabilité sociale au changement climatique. Cette nouvelle tendance a émergé principalement pour deux raisons. Tout d'abord, de sérieux doutes concernant l'existence effective d'un changement climatique ont pu être dissipés et ses conséquences sont devenues de plus en plus tangibles avec le temps. La température mondiale augmente encore plus vite que prévu ${ }^{4}$ et depuis le milieu du XXe siècle, le nombre de cas déclarés de catastrophes naturelles n'a cessé d'augmenter, passant d'environ 30 en 1960 à 205 en 1980 et à 413 en 2000. Le nombre de personnes concernées durant la même période est passé d'environ 50 millions en 1970 à une moyenne annuelle de 227,5 millions entre 2000 et 20095. Ensuite, il est devenu évident que les efforts en matière d'atténuation du changement climatique dans le cadre de la politique climatique actuelle et les négociations hésitantes qui l'accompagnent ne suffiraient pas. Par conséquent, l'adaptation ne pouvait plus être vue comme un exercice superflu ou lointain ${ }^{6}$.

Malgré ce changement, le discours sur le climat reste encore profondément enraciné dans la problématique formulée par les études P\&E. Cela s'explique par le fait que la discipline fut fondée en 1798 par Thomas Malthus (1766-1834) et prolongée au XXe siècle par le néo-malthusianisme (courant représenté notamment par le Club de Rome, créé en 1968) ${ }^{7}$. Alors que Malthus était préoccupé par le déséquilibre entre la croissance exponentielle

3. Bien que l'adaptation joue un rôle dans les rapports principaux, elle n'est pas ou seulement marginalement prise en compte dans les résumés destinés aux décideurs (voir GIEC AR1, WG III, 1990 ; I. GIEC AR2, le GT II, 1996 ; GIEC AR2, WG III, 1996, pp. 1-16). En revanche, le troisième rapport du GIEC distingue pour la première fois l'atténuation et la vulnérabilité/adaptation en confiant ces thématiques à différents groupes de travail, ce qui fait de l'adaptation une nouvelle priorité. Ceci est clairement reflété dans le "Résumé pour décideurs » qui préconise l'élaboration d'outils d'évaluation des risques et des stratégies d'adaptation (GIEC AR3, le GT II, 2001, p. 17, et GIEC AR4, Groupe de travail II, 2007, pp. 7-22).

4. Lean J., Rind D. "How will Earth's Surface Temperature Change in Future Decades?", Geophysical Research Letters, vol. 36, L15708, 2009, p. 5.

5. Les chiffres sont compilés à partir de EM-DAT, Number of disasters reported 1900-2010 and Number of people reported affected by natural disasters 1900-2010, Natural Disaster Trends of the International Disaster Database, Centre for Research on the Epidemiology of Disasters - CRED, http://www.emdat.be/natural-disasters-trends ; EM-DAT, 2010 Disasters in Numbers, CRED, http://cred.be/sites/default/files/PressConference2010.pdf ; Guha-Sapir D., Vos F., Below R., Ponsere S., Annual Statistical Review 2010: The Numbers and Trends, Brussels, CRED, mai 2011, p. 21, http://www.cred.be/sites/default/files/ADSR_2010.pdf (pages consultées le 21 février 2011). Bien que l'augmentation des chiffres puisse s'expliquer en partie par l'amélioration des rapports sur les catastrophes et la croissance démographique dans les régions à risque, elle ne peut être réduite à ces facteurs.

6. Hunter D., "Human rights implications for climate change negotiations", Oregon Review of International Law, vol. 11, n², 2009, p. 359. En 2007, le GIEC déclarait que l'adaptation allait être inévitable (IPCC AR4, WG II, op. cit., p. 747).

7. Pour une présentation sur les approches P\&E, voir Sherbinin A., Carr D., Cassels S., Jiang L., "Population and Environment", Annual Review of Environment and Resources, vol. 32, 2007, pp. 345-373. Pour une synthèse du néo-malthusianisme sur les « récits de la dégradation ", voir Hartmann B., "Rethinking the Role of Population in Human Security", in Matthew R., Barnett J., McDonald B., O’Brian K. (eds.), Global Environmental Change and Human Security Massacbusetts, Massachusetts Institute of Technology, MIT Press, 2010, pp. 193-214. 
de la population et l'augmentation présumée linéaire de la production alimentaire (mettant l'accent sur la variable « sécurité alimentaire »), le néo-malthusianisme se réfère à la pression démographique sur les ressources naturelles et la dégradation de l'environnement en général en mettant en avant la variable écologique. L'augmentation de la population et la consommation croissante ont toujours été, et sont encore, les principaux déterminants pour conceptualiser les pressions exercées sur les ressources. En raison des connotations alarmistes des discours tenus par cette école de pensée, John Dryzek la qualifie de «survivalism 8 ». Dans le même temps, l'approche néo-malthusienne et d'autres courants au sein des études P\&E mettent fortement l'accent sur les solutions technologiques 9 .

Les recherches sur le changement climatique d'origine anthropique renforcent la pensée néo-malthusienne à plusieurs titres. L'analyse des impacts de premier et de deuxième ordre tout comme les projections climatiques effectuées sont clairement liées aux concentrations de GES générés par une consommation non durable de l'énergie (combustibles fossiles en particulier) et des ressources naturelles qui conduit à la déforestation, à une nouvelle utilisation des terres et à la disparition des puits de carbone ${ }^{10}$. Les politiques d'atténuation mettent l'accent sur des instruments et des approches technologiques fondés sur l'économie de marché. La consommation d'énergie fossile doit diminuer au profit d'énergies renouvelables via le développement de technologies d'économie d'énergie et d'incitations du marché à maintenir et accroître les puits de carbone. Des systèmes d'échange de droits d'émission sont établis, qui entraînent des mécanismes élaborés de comptage du carbone émis dans l'atmosphère ${ }^{11}$. La « comptabilité carbone » est l'un des piliers de la CCNUCC 12 .

8. Dryzek J., The Politics of the Earth: Environmental Discourses, New York, Oxford University Press, 2e édition, 2005. Voir en particulier le chapitre "Looming Tragedy: Survivalism”.

9. Voir Sherbinin A., Carr D., Cassels S., Jiang L., "Population and Environment", op. cit., p. 348. Ceci est très clairement exprimé dans la formule IPAT du Club de Rome qui définit « l'impact sur l'environnement » en relation avec la « population », la « richesse » et la « technologie».

10. Le fait que l'amplitude de la consommation des ressources soit liée à la taille de la population figure, par exemple, dans le rapport spécial du GIEC sur les scénarios d'émissions (SRES) qui utilise la croissance de la population comme une des variables sur lesquelles est fondée l'argumentation. En plus de la «population ", les scénarios considèrent aussi les variables « monde » (à travers l'interdépendance entre les régions et les nations), « économie », « gouvernance » et «technologie».

11. Les chercheurs qui mettent explicitement l'accent sur les émissions de GES prennent également en considération, pour modéliser la consommation d'énergie, une variété de facteurs démographiques tels que la pyramides des âges, le taux d'urbanisation, la taille des ménages ou le degré de mobilité (Sherbinin A., Carr D., Cassels S., Jiang L., "Population and Environment”, op. cit., p. 359f). Les scénarios SRES se réfèrent, au moins en partie, à ces déterminants (voir note 5), mais sans nécessairement étudier le lien complexe entre ces déterminants et le niveau d'émissions

12. Hunter D., "Human rights implications for climate change negotiations", op. cit., p. 340. 
Les analyses des impacts de troisième ordre renforcent le récit de la « survie » en pointant la perte extraordinaire de sols fertiles due à la désertification, l'érosion, l'élévation du niveau des mers, l'augmentation de la variabilité des précipitations... Ces analyses mènent aux impacts de quatrième ordre comme, par exemple, ceux sur les récoltes, l'approvisionnement en eau et le nombre croissant des lieux d'habitation susceptibles d'être déplacés, abandonnés ou de s'effondrer. L'adaptation envisagée pour éviter la souffrance humaine s'appuie fortement là aussi sur des solutions technologiques et des techniques de gestion des ressources (comme la gestion de l'eau par la construction de barrages, l'irrigation et les travaux pour sécuriser la production agricole ou encore les nouvelles règles d'aménagement et de construction pour rendre les établissements humains plus résistants). L'objectif final est d'assurer la viabilité des sociétés locales dans un environnement naturel en mutation.

\section{L'influence des études P\&E sur les recherches en matière de migrations climatiques}

Le thème de la migration climatique s'est trouvé classé au chapitre des impacts de quatrième ordre. Il n'est pas surprenant qu'il soit de nouveau abordé, dans une large mesure, à travers les hypothèses des études P\&E, en particulier celles portant sur la menace de pénurie de ressources. En fait, indépendamment du discours sur le climat, le cadre analytique des études P\&E a fourni des récits qui ont été recyclés pour interroger le lien entre environnement et migration. En 1990, le rapport AR1 du GIEC adoptait déjà cette perspective analytique pour les questions migratoires ${ }^{13}$. Celle-ci peut être résumée comme suit ${ }^{14}$ : la dégradation de l'environnement et la pression démographique génèrent des migrations et des réfugiés environnementaux ${ }^{15}$; le déplacement (en particulier des pauvres) aux abords des forêts conduit à la déforestation ${ }^{16}$; la migration entraîne aussi des conflits (ethniques) autour de l'appropriation des maigres ressources renouvelables ainsi que des émeutes urbaines dans les lieux de destination ${ }^{17}$.

13. IPCC AR1, WG II, chapitre 5, 1990.

14. En se basant sur Hartmann B. ("Rethinking the Role of Population in Human Security", op. cit., p. 197f) et Sherbinin A., Carr D., Cassels S., Jiang L. ("Population and Environment”, op. cit., p. 351), Vaclav Smil propose la séquence suivante $:$ "l'érosion des terres en pente = réfugiés environnementaux $=$ villes surpeuplées $=$ instabilité politique $=$ violence $»($ Smil V., “China’s Evnironment and Security: Simple Myths and Complex Realities”, SAIS Review, vol. $17, \mathrm{n}^{\circ} 1, \mathrm{p} .108$.

15. Pour une analyse des racines malthusiennes de la notion de "réfugié environnemental », voir Saunders P., "Environmental Refugees: The Origins of a construct”, in Scott P., Sullivan S. (eds.), Political Ecology, Londres, Arnold, 2000, pp. 218-246. Par exemple, le PNUD a suivi cette ligne de pensée dans son rapport de 1994 sur le développement humain.

16. Voir par exemple Geist H., Lambdin E., What Drives Tropical Deforestation? A Meta-analysis of Proximate and Underlying Causes of Deforestation Based on Sub-National Case Study Evidence, LUCC Report Series, $\mathrm{n}^{\circ}$ 4, Louvain-la-Neuve, LUCC International Project Office, 2001.

17. Le représentant le plus éminent en est Thomas Homer-Dixon avec son livre Environment, Scarcity and Violence, Princeton, Princeton University Press, 1999. 
Le débat actuel sur les migrants climatiques se compose de cinq volets principaux qui, tous, supposent la présence d'un lien (plus ou moins) direct entre changement climatique et migration et partagent l'hypothèse que ce lien découle de la raréfaction des ressources. La perte des terres due à l'élévation du niveau de la mer, la désertification et les glissements de terrain ou encore le stress hydrique conduisent à la privation des moyens de subsistance essentiels, et obligent ainsi les gens à quitter leur lieu de vie ${ }^{18}$. Cela peut se produire suite à des événements soudains ou progressivement, en raison de changements lents mais irréversibles. Cette situation peut également résulter de l'affaiblissement des capacités d'adaptation en raison de l'augmentation de la fréquence d'événements météorologiques extrêmes qui ne permettent pas le rétablissement des communautés touchées.

Ces cinq volets du débat sont les suivants: le volet « écosystémique », le volet « conflits », le volet « réfugiés », le volet « adaptation » et le volet « réinstallation ». Le volet « écosystémique » constitue l'étincelle initiale d'où découlent les autres fils d'argumentation. L'argument dominant est de considérer la cause climatique comme le principal facteur de la migration forcée ${ }^{19}$. Cela conduit généralement à l'identification de zones à haut risque et à des projections du nombre de personnes risquant d'être déplacées ${ }^{20}$. Le chiffre le plus cité à cet égard est celui avancé en 1996 par Norman Myers de 200 millions de réfugiés environnementaux d'ici à 2050 (et actualisé en 2007 à 250 millions de personnes) ${ }^{21}$. Ces chiffres ont été cités dans de nombreux documents portant sur le changement climatique tels que les rapports du GIEC, le rapport Stern, les documents de l'ONU, etc. Ces projections sur l'augmentation extraordinaire de migrations climatiques sont généralement expliquées par la

18. Voir par exemple Oliver-Smith A., "Climate Change and Population Displacement", in Cate S., Nuttall M. (eds.), Anthropology and Climate Change: From Encounters to Action, Walnut Creek, Left Coast Press, 2009, p. $117 f$.

19. Pour une catégorisation des facteurs climatiques voir inter alios Hugo G., Migration, Development and Environment, OIM, Migration Research Series, $\mathrm{n}^{\circ}$ 35, Genève, OIM, 2008 ; Kniveton D., Schmidt-Verkerk K., Smith Ch., Black R., Climate Change and Migration: Improving Methodologies to Estimate Flows, OIM, Migration Research Series, $n^{\circ}$ 33, Genève, OIM, 2008 ; Renaud F., Bogardi J. J., Dun O., Warner K., Control, Adapt or Flee How to Face Environmental Migration?, InterSecTions, Interdisciplinary Security Connections, $\mathrm{n}^{\circ}$ 5, Bonn, United Nations University - Environment and Human Security (UNU-EHS), 2007 ; ainsi que le récent numéro spécial "Migration and Global Environmental Change - Review of Drivers of Migration”, Global Environmental Change, vol. $21, n^{\circ} 1,2011$.

20. Pour une présentation des différentes estimations et du débat sur l'existence ou non de liens entre ces phénomènes, voir Gemenne F., "Why the Numbers Don't Add Up: A Review of Estimates and Predictions of People Displaced by Environmental Change", Global Environmental Change, vol. 21, $\mathrm{n}^{\circ} 1,2011$, pp. S50-S58.

21. Pour le chiffre de 200000 réfugiés, voir Myers N., "Environmentally-Induced Displacements: the State of the Art", présentation au Colloque international Environmentally-Induced Population Displacements and Environmental Impacts Resulting from Mass Migration, Genève, OIM et Haut-Commissariat des Nations unies pour les réfugiés (UNHCR), 21-24 avril 1996. Pour le chiffre de 250000 réfugiés, voir The Human Tide: The Real Migration Crisis, A Christian Aid Report, mai 2007, p. 48 (http://www.christianaid.org.uk/images/human-tide.pdf, consulté le 22 février 2011). 
détérioration des services écosystémiques essentiels. Elles sont une exhortation à agir pour que le changement climatique soit freiné, voire arrêté. Certains considèrent ce volet du débat comme une stratégie de lobbying des environnementalistes pour augmenter la pression sur les décideurs afin de renforcer les mesures d'atténuation du changement climatique ${ }^{22}$.

Sur la base de ces projections alarmantes, les chercheurs travaillant sur la paix et les conflits ainsi que les institutions concernées par les questions de sécurité débattent du risque de voir émerger des conflits en raison des migrations de masse induites par le changement climatique ${ }^{23}$. Dans cette perspective, la migration est considérée comme l'une des diverses menaces à la sécurité auxquelles les sociétés doivent faire face sur une planète qui se réchauffe inexorablement, au même titre que les contentieux territoriaux, les conflits inter-étatiques ou ceux liés à l'appropriation des ressources au sein d'un même État. Ce débat souligne également l'importance de la dimension « ressources » du problème en lui ajoutant une dimension « conflit ». Il dérive plus ou moins directement des travaux P\&E de Homer-Dixon sur la dégradation de l'environnement et les conflits ${ }^{24}$. L'événement politique le plus significatif et pertinent à cet égard est la discussion qui a eu lieu au sein du Conseil de sécurité des Nations Unies en 200725.

Le troisième volet du débat porte sur la sécurité humaine et les questions de gouvernance. Les acteurs, surtout des universitaires et des ONG, appellent à l'instauration d'un nouveau statut pour lesdits « réfugiés climatiques » et/ou

22. McGregor J., "Climate Change and Involuntary Migration: Implications for Food Security", Food Policy, vol. 19, n², 1994, p. 127. Voir aussi Gemenne, "Why the Numbers Don’t Add Up: ...”, op. cit., p. 9.

23. Voir les différentes contributions in Nordås R,, N. P. Gleditsch (eds.), "Climate Change and Conflict”, Political Geography, vol. 26, n 6, 2007, pp. 639-655. Les auteurs de ce numéro sont, parmi d'autres, J. Barnett et W. Adger, R. Reuveny, J. D. Fearon et D. D. Laitin, C. Raleigh et H. Urdal. Voir aussi Clark W., Environmentally Induced Migration and Conflict, external expertise for the German Advisory Council on Global Change (WBGU) Flagship Report World in Transition: Climate Change as a Security Risk, Berlin, 2007. Le potentiel de conflit lié aux migrations climatiques est également souligné par le père des études sur l'environnement et les conflits, Homer-Dixon ("Terror in the Weather Forecast", New York Times, 24 avril 2007). En bref, la question a déjà été soulevée dans le 2e rapport d'évaluation du GIEC en référence à Homer-Dixon T., Boutwell J. H., Rathjens G. W., "Environmental change and violent conflict”, Scientific American, vol. 268, n² 2, 1993, pp. 38-45 et Shurke A., "Pressure points: Environmental Degradation, Migration, and Conflict", Occasional Paper Series of the Project on Environmental Change and Acute Conflict, $\mathrm{n}^{\circ} 3$, Toronto, University of Toronto, 1993 (IPCC 1996, WG II, p. 496).

24. Homer-Dixon T., Environment, Scarcity and Violence, op .cit.

25. UNSC, Security Council holds First-Ever Debate on Impact of Climate Change on Peace, Security, Conseil de sécurité 5663e Meeting, SC/9000, avril 2007. L’OIM a également considéré cette question dans sa 94e session (OIM, Discussion Note: Migration and the Environment, MC/INF288, novembre 2007). L'action du CSNU a été suivie par une résolution de l'Assemblée générale des Nations Unies et un rapport du Secrétaire général à l'Assemblée générale des Nations Unies (UNGA, Climate Change and its Possible Security Implications, résolution adoptée par l'Assemblé générale, 63/281, juin 2009 ; UNGA, Climate Change and its Possible Security Implications, Rapport du Secrétaire général, A/64/350, septembre 2009). 
à la révision et l'ajustement des conventions internationales existantes ${ }^{26}$. La proposition la plus connue d'un nouveau statut pour les réfugiés climatiques est celle de Frank Biermann et Ingrid Boas, qui plaident pour un statut de groupe (par opposition à un droit individuel) et se concentrent sur des cas qui, selon les connaissances scientifiques actuelles, peuvent être clairement attribués à des changements environnementaux liés à des modifications climatiques ${ }^{27}$. Ici, l'hypothèse selon laquelle le climat constitue le principal facteur (push factor) de déplacement sert clairement de point de départ. Le naufrage de petits États insulaires en fournit la preuve. Les discussions sur les réfugiés climatiques sont cependant très diversifiées, allant de l'analyse juridique des instruments actuellement disponibles à des propositions de nouvelles politiques publiques ${ }^{28}$. Les tentatives pour obtenir une réforme de la Convention de Genève ou un régime juridique distinct pour les réfugiés climatiques (comme celui du droit d'asile) ont échoué jusqu'ici. En revanche, la proposition de Biermann et Boas semble toujours d'actualité en raison de sa proximité avec le cinquième volet ${ }^{29}$.

Le quatrième volet du débat va au-delà des questions relatives au déplacement imminent des populations, et considère la migration comme un potentiel, comme un moyen d'adaptation au changement climatique ${ }^{30}$. Ce nouveau récit découle des discours sur les liens entre développement et migrations et

26. WBGU, World in Transition: Climate Change as a Security Risk, op. cit., p. 129.

27. Biermann F., Boas I., "Preparing for a Warmer World: Towards a Global Governance System to Protect Climate Refugees”, Global Governance Working, n³3, novembre 2007.

28. Pour les instruments juridiques, voir par exemple Ammer M., Climate Change and Human Rights: The Status of Climate Refugees in Europe, Ludwig Boltzmann Institute of Human Rights, juin 2009 ; Ammer M., Nowak M., Stadlmayr L., Hafner G., Rechtsstellung und rechtliche Behandlung von Umweltflüchtlingen, Umweltbundesamt, $\mathrm{n}^{\circ}$ 54, 2010. Pour des propositions de politiques publiques, voir Biermann F., Boas I., "Preparing for a Warmer World: Towards a Global Governance System to Protect Climate Refugees”, op. cit. ; voir aussi Docherty B., Tyler G., "Confronting a Rising Tide: A Proposal for a Convention on Climate Change Refugees", Harvard Environmental Law Review, vol. 33, n 2, 2009, pp. 349-403. Pour le naufrage de États insulaires, voir par exemple McAdam J., "Disappearing States, Statelessness and the Boundaries of International Law”, in McAdam J. (ed.), Climate Change and Displacement: Multidisciplinary Perspectives, Oxford (Portland), Hart Publishing, 2010, pp. 105-130. Dans ce livre, voir en particulier Zetter R., "Protecting People Displaced by Climate Change: Some Conceptual Challenges”, pp. 131-150 (sur les personnes déplacées internes) et Penz P., "International Ethical Responsibilities to 'Climate Change Refugees”, pp. 151-174 (sur les questions de compensation et autres questions juridiques). Voir aussi la contribution de Cournil Ch., "The Protection of 'environmental refugees' in international law”, pp. 359-387, et celle de Epiney A., “Environmental refugees: Aspects of international state responsibility”, in Piguet E., Pécoud A., Guchteneire P. (eds.), Migration and Climate Change, Cambridge, Cambridge University Press, UNESCO, 2011, pp. 388414.

29. Voir le résumé proposé par Bauer S., Climate Refugees beyond Copenhagen: Legal concept, political Implications, Diakonisches Werk der EKD e.v. for 'Brot für die Welt', Stuttgart, mars 2010, p. 21f.

30. Une analyse de ce volet est proposé par Barnett J., Webber M., "Migration as Adaptation: opportunities and Limits”, in McAdam (ed.), op. cit., p. 37-56. La question de l'envoi de fonds comme moyen d'adaptation au changement climatique est particulièrement développée dans l'étude du Vietnam proposée par Adger N., Kelly M., Winkels A., Huy L.Q., Locke C., "Migration, Remittances, Livelihood Trajectories, and Social Resilience", Ambio, 4-31, juin 2003. 
des recherches sur les migrations qui soulignent l'importance des envois de fonds comme stratégie de diversification des revenus et de protection contre les risques ${ }^{31}$. Le facteur humain refait ainsi surface dans la prise des décisions en matière migratoire. Cependant, cette idée est immédiatement convertie en un outil politique, soit pour investir les fonds dans l'adaptation in situ, soit pour rendre les ménages déplacés moins dépendants des ressources naturelles sur leur lieu d'habitation. Ici, les envois de fonds sont censés pallier le problème de la rareté des ressources. En conséquence, l'Organisation internationale pour les migrations préconise désormais des programmes de migrations planifiées du travail. La migration contrôlée devient ainsi, tout d'un coup, une partie de la solution du problème climatique ${ }^{32}$.

Enfin, le cinquième volet du débat sur les migrants climatiques consiste en une mesure préventive pour éviter le déplacement non contrôlé : la réinstallation des communautés vulnérables ${ }^{33}$. Cette «solution » répond partiellement au problème de la dégradation des ressources et cherche à le résoudre par la réinstallation des personnes dans des lieux aux conditions plus sûres et viables. La réinstallation est considérée comme la seule mesure envisageable pour protéger ceux qui vivent dans des endroits où aucune adaptation in situ est impossible. Couplé avec le quatrième volet, ce cinquième volet permet d'éviter une migration de masse incontrôlée perçue comme une forme de «maladaptation 34 ». Les mesures de migration ordonnée et de réinstallation ont même trouvé leur place dans les négociations de la CCNUCC. En décembre 2010, lors de la COP-16 à Cancun, le Groupe de travail spécial sur l'action concertée à long terme au titre de la CCNUCC (AWG-LCA) a inclus pour la première fois la question du déplacement, de la migration et du déplacement pla-

31. Pour une présentation des liens entre développement et migrations voir Faist T., "Migrants as Transnational Development Agents: An Inquiry into the Newest Round of the MigrationDevelopment Nexus", Population, Space and Place, 1-14, 2008, pp. 21-42. Pour la fonction de protection contre les risques par l'envoi de fonds voir Stark O., Levhari D., "On migration and risk in LDCs", Economic Development and Cultural Change, vol. 31, n ${ }^{\circ} 1,1982$, pp. 191196.

32. Moor N., Labour Migration for Vulnerable Communities: A Strategy to Adapt to a Changing Environment, Center on Migration, Citizenship and Development, COMCAD, Working Paper $n^{\circ}$ 101, Bielefeld University, 2011.

33. La réinstallation préventive est particulièrement discutée dans le cas des îles menacées par la hausse du niveau des mers. Voir par exemple Campbell J., "Climate-Induced Community réinstallation in the Pacific: The Meaning and Importance of Land", in McAdam (ed.), op. cit., pp. 57-80 ; Ferris E., Cernea M., Petz D., On the Front Line of Climate Change and Displacement: Learning from and with Pacific Countries, Brookings Institution/London School of Economics, septembre 2011 ; Boege V., Challenges and Pitfalls of Resettlement Measures: Experiences in the Pacific Region, Center on Migration Citizenship and Development, COMCAD Working Paper $n^{\circ} 102$, Bielefeld University, 2011. La réinstallation planifiée suite à une dégradation de l'environnement ou une catastrophe naturelle a également fait l'objet de trois études de cas du programme EACH-FOR, à savoir Zhang Q., Inner Mongolia Case Study Report, 2009; Stal M., Mozambique Case Study Report, 2009 ; et Dun O., Vietnam Case Study Report, 2009 (www.each-for.eu/index.php?module= d_documents, consulté le 21 février 2011).

34. UNGA, Climate Change and its Possible Security Implications, Rapport du Secrétaire général, A/64/350, septembre 2009, p. 7. 
nifié dans son document final (ce que l'on appelle le « Résultat LCA ») ${ }^{35}$. Ceci est considéré comme une grande réussite sur le plan politique parce que les autres tentatives pour inclure une référence au défi de la migration climatique au sein d'un accord officiel avaient jusqu'alors échoué ${ }^{36}$.

Parallèlement à ces évolutions, des critiques ont commencé à se faire entendre à propos des postulats imposés par les études P\&E sur le débat en matière de migrations climatiques et ses connotations alarmistes. Les chercheurs critiques - principalement des spécialistes des migrations - sont souvent qualifiés de «sceptiques " 37 . Cependant, force est de reconnaître que ce scepticisme à l'égard du débat sur les « migrants climatiques » ne concerne pas le changement climatique ou ses conséquences graves sur les conditions de subsistance. Il ne consiste pas non plus en une réfutation généralisée des solutions techniques pour faire face aux problèmes de la rareté des ressources. Du point de vue scientifique, la principale préoccupation de ces chercheurs tient plutôt au fait que la réalité du lien entre climat et migration n'est pas décrite avec précision (il s'agirait d'une inconsistance scientifique). Notre point de vue, développé plus loin, est que sur le plan politique, des conclusions hâtives et des solutions rapides pourraient s'avérer contre-productives.

Des chercheurs engagés dans des travaux empiriques sur les migrations et les réfugiés ont analysé des études de cas pour prouver qu'aucune causalité simple ne peut être établie entre le changement climatique et les migrations ${ }^{38}$. Intentionnellement ou non, ces efforts disqualifient l'argument dominant au sein des études P\&E selon lequel le facteur déterminant (du point de vue scientifique) ou le problème principal (du point de vue politique) est le climat. Les principaux contre-arguments avancés sont de différents ordres : les processus migratoires ont des causes multiples et d'autres facteurs, sociaux et économiques, interviennent ou même prévalent ; un contexte dégradé pourrait également conduire à une diminution des migrations en raison de l'impossibi-

35. UNFCCC, The Cancun Agreements: Outcome of the work of the Ad Hoc Working Group on Long-term Cooperative Action under the Convention, Decision 1/CP.16, rapport de la seizième session de la Conférence des Parties, tenue à Cancun du 29 novembre au 10 décembre 2010, Addendum, FCCC/CP/2010/7/Add.1, mars 2011, Para 14(f).

36. Mayer B., Climate Migrants and the IOM at Cancun Conference on Climate Change, janvier 2011 (http://oppenheimer.mcgill.ca/Climate-Migrants-and-the-IOM-at?llang=en, consulté le 21 février).

37. Pour une discussion détaillée de la position des chercheurs « alarmistes » (par opposition aux chercheurs «sceptiques »), voir Gemenne F., "How They Became the Human Face of Climate Change. Research and Policy Interactions in the Birth of the 'Environmental Migration’ Concept”, in Piguet E., Pécoud A., Guchteneire P. (eds.), op. cit., pp. 225-259.

38. K. Schmidt-Verkerk propose une comparaison intéressante de deux cas similaires en ce qui concerne leurs conditions climatiques mais différents en termes de comportement migratoire des populations : Schmidt-Verkerk K., “Buscando la Vida” - How Do Perceptions of Increasingly Dry Weather Affect Migratory Behaviour in Zacatecas, Mexico?”, in Affifi T., Jäger J. (eds.), Environment, Forced Migration and Social Vulnerability, Londres, Springer, 2010, pp. 99-116. Pour un bon aperçu des éléments empiriques concurrents, voir Kinveton D., Schmidt-Verkerk K., Smith C., Black R., Climate Change and Migration: Improving Methodologies to Estimate Flows, OIM, Migration Research Series, n 33, 2008, pp. 32-37. 
lité d'assumer les coûts de la mobilité ; la migration est un mécanisme traditionnel d'adaptation impliquant un choix humain et non pas nécessairement un déplacement forcé ; la migration est sélective, tous les membres d'une communauté ne peuvent ou ne veulent se déplacer ${ }^{39}$. En ce qui concerne le quatrième volet, l'approche politique qui en découle ignore qu'en dépit des envois de fonds, l'accélération du processus de migration a dans le même temps des répercussions négatives sur la résilience des communautés d'origine en raison des changements dans la composition démographique. Sur ce point, les recherches sur la relation entre migration, démographie et capacité d'adaptation se multiplient ${ }^{40}$.

Jusqu'ici, cependant, ces recherches empiriques n'ont pas encore reçu la même attention dans le discours politique que les cinq principaux volets du débat. En ce qui concerne les trois premiers volets, cela peut s'expliquer par l'accent alarmiste qui attire facilement l'attention du public. Quant aux quatrième et cinquième volets, en présentant la migration non plus comme un problème mais comme une solution au changement climatique, ils rendent les décideurs plus sensibles à l'enjeu migratoire ${ }^{41}$. Le fait que la migration, le déplacement et la réinstallation figurent dans le document final du LCA de la CCNUCC en constitue la preuve. La réinstallation est même en train de devenir un point central dans la poursuite du débat. Mais qu'est-ce que cela signifie sur le plan politique ? Certains pourront trouver cela anecdotique mais cela signifie plusieurs choses : tout d'abord que le « migrant climatique » se voit intégré dans un régime juridique disposant de fonds considérables; ensuite que le débat s'inscrit dans un cadre dominé par les discours des études P\&E et la croyance en la pertinence de solutions techniques; et enfin que le facteur humain pourrait de nouveau refaire surface. Dans cette perspective, il est à craindre que la réinstallation (cinquième volet) gagne en importance en tant que politique d'adaptation. La question se pose donc de savoir comment la réinstallation en tant qu'adaptation peut se faire sans nuire davantage à la population touchée ? Le cadre de la CCNUCC révèle en outre très clairement ce sur quoi nous aimerions insister plus particulièrement : qu'en est-il des personnes déplacées par les mesures d'adaptation et d'atténuation ? En effet, plu-

39. Black propose un résumé des principaux points de critique dans Black R., Environmental Refugees: Myth or Reality?, UNHCR, New Issues in Refugee Research, Working Paper $\mathrm{n}^{\circ}$ 34, mars 2001. Également Castles S., Environmental Change and Forced Migration: Making Sense of the Debate, UNHCR Issues, Refugee Research Working Paper n 70, 2002.

40. McLeman propose également un examen approfondi de la bibliographie existante dans McLeman R., "Impacts of Population Change on Vulnerability and the Capacity to Adapt to Climate Change and Variability: A Typology Based on Lessons From a Hard Country", Population and Environment, vol. 31, n ${ }^{\circ}$ 5, 2010, pp. 286-316. Des nouvelles études empiriques de terrain seront présentées par McLeman R., Ford J., "How Demographic Change and Migration Influences Adaptation to Climate Change: Examples from Rural Eastern Ontario and Nunavut, Canada", in Faist T., Schade J. (eds.), Disentangling Migration and Climate Change: Toward an Analysis of Concepts, Methodologies, and Policies, Dordrecht, Springer 2013 (à paraître).

41. Voir Vlassopoulos C., "Defining Environmental Migration in the Climate Change Era: Problem, Consequence or Solution?” in Faist T., J. Schade (eds.), ibid. 
sieurs politiques climatiques entrainent implicitement des réinstallations. Ainsi, le cinquième volet tend actuellement vers un sixième volet du débat portant sur les migrants climatiques : le volet «politique climatique».

\section{Un nouveau défi : politique climatique et migration forcée}

Jusqu'en 2011, la question de savoir dans quelle mesure le changement climatique, mais aussi les politiques du changement climatique génèrent des déplacements et des migrations forcées n’a pas été, à quelques exceptions près, un thème majeur dans le discours sur les migrations climatiques ${ }^{42}$. En effet, la proposition de Biermann et Boas de créer une Convention sur les réfugiés climatiques a explicitement exclu, par exemple, « la migration forcée provoquée par des mesures d'atténuation et d'adaptation au réchauffement de la planète, y compris la construction de barrages [...] et [...] les grandes plantations de bio-carburants ", parce que les auteurs considéraient que l'impact de ces mesures devait être principalement attribué à une gouvernance médiocre au niveau local et national ${ }^{43}$. Cette position a toutefois significativement changé depuis que la réinstallation a été incluse dans le document final du LCA et a donc trouvé un point d'entrée dans le régime de la CCNUCC. En effet, 2011 a connu une prolifération des contributions universitaires mettant en exergue toutes formes possibles de réinstallation dans le cadre de la CCNUCC. Ces travaux ont porté à la fois sur la réinstallation comme mesure d'adaptation, la réinstallation due à d'autres mesures d'adaptation, la réinstallation due à des mesures d'atténuation, ainsi que les déplacements provoqués par l'échec d'une réinstallation ${ }^{44}$. Dans ce contexte, un nouveau groupe d'experts a émergé dans le domaine des migrations climatiques : les experts en réinstallation ${ }^{45}$.

42. Parmi ces exceptions : Schade J., "Climate Change and the Challenge of Organized Resettlement", présentation à la conférence Protection and Reparations for 'Climate Refugees': Imperatives \& Options, Brot für die Welt with Oikoumene Pacific and World Council of Churches, Chavannes-de-Bogis, 3-4 mai 2010 (www.oikoumene.org/fileadmin/files/wcc-main/documents/p 4/climate/ Compendium\%20final.pdf, consulté le 21 février 2011); Schade J., communication à la 1 ère conférence ESF sur les migrations environnementales (voir Faist T., "Environmental Change and Migration: From Vulnerabilities to Capabilities", Rapport de la Conférence ESF-ZiFBielefeld, Research Conference, Bad Salzuflen, 5-9 décembre 2010, p. 9, www.uni-bielefeld.de/tdrc/ag_comcad/downloads/envimig_esf_conference-report.pdf, consulté le 21 février 2011); Gemenne F., présentation à la conférence sur les règlements internationaux, octobre 2010 (voir Koppel Maldonado J., "A New Path Forward: Researching and Reflecting on Forced Displacement and Resettlement Report on the International Resettlement Conference: Economics, Social Justice, and Ethics in Development-Caused Involuntary Migration, the Hague, 4-8 October 2010”, Journal of Refugee Studies, vol. 25, n 2, 2012, pp. 193-220).

43. Pour rendre leur proposition plus réaliste, ils ont aussi exclu les cas de migrations et de déplacements résultant d'autres types de dégradation de l'environnement, tels que les accidents industriels, les éruptions volcaniques ou les conflits résultant d'une dégradation environnementale étant donné qu'ils peuvent être pris en main par d'autres institutions. Biermann F., Boas I., "Preparing for a Warmer World: Towards a Global Governance System to Protect Climate Refugees", op. cit., p. 4.

44. Sherbinin A., Castro M., Gemenne F., Cernea M. M., Adamo S., Fearnside P. M., Krieger G., Lahmani S., Oliver-Smith A., Pankhurst A., Scudder T., Singer B., Tan Y., Wannier G., 
Malheureusement, la réinstallation n'est en rien une tâche facile. Il est devenu évident à partir des évictions produites par des projets de développement que celles-ci impliquent des risques élevés pour les personnes concernées - et ceci même lorsqu'un projet comporte un plan de réinstallation planifiée. L'expérience et la littérature scientifique montrent que les réinstallations comportent des risques élevés en termes de bien-être des personnes. Il est intéressant de constater que le chiffre souvent cité d'un milliard de réfugiés environnementaux d'ici à 2050 comprend en effet 645 millions de personnes potentiellement déplacées par des projets de développement qui s'ajoutent aux 250000 personnes évoquées dans le rapport Christian Aid et aux autres réfugiés générés par des conflits liés à la rareté des ressources ${ }^{46}$. Cela signifie que les expulsions dues à des projets de développement pourraient s'avérer être un plus grand défi que les déplacements consécutifs à des catastrophes naturelles.

Les chiffres sont impressionnants. Par exemple, la construction de barrages en Chine a déplacé plus de 10 millions de personnes entre 1959 et 1989 47. Dans la seconde moitié du XXe siècle, 45,1 millions de personnes ont été déplacées par des projets de développement en Chine ${ }^{48}$. L'Inde compte environ 50 millions de personnes déplacées en raison de projets de développement réalisés depuis le milieu du XXe siècle. Plus de 21 millions d'entre elles ont été chassées suite à la construction de barrages ${ }^{49}$. Les consultants de la Banque mondiale estiment que durant les seules années 1990, entre 90 et 100 millions de personnes ont été délogées au niveau mondial en raison de programmes d'infrastructures (barrages, urbanisation, routes, etc.) ${ }^{50}$. Ainsi, de nombreuses personnes sont déplacées à cause des projets de développement en dépit du fait que la plupart sont théoriquement accompagnés par des

Boncour P., Ehrhart C., Hugo G., Pandey B., Shi G., "Preparing for Resettlement Associated with Climate Change”, Science, vol. 334, n 6065, 2011, pp. 456-457 ; Reilly M., Hossain Y., The Impact of Low-Carbon Policy on Migration, DR15 of the UK Government's Foresight Project "Migration and Global Environmental Change", octobre 2011, p. 27ff. ; McDowell C., Climate Change Adaptation and Mitigation: Implications for Land Acquisition and Population réinstallation, WP3 pour le projet Foresight du gouvernement britanique "Migration and Global Environmental Change", octobre 2011 ; Ferris E., "Planned reinstallations, Disasters and Climate Change", présentation à la Conference on Climate Change and Migration in the Asia-Pacific: Legal and Policy Response, University of New South Wales, Sydney, 10 novembre 2011.

45. Parmi eux, certains des co-auteurs de Sherbinin et al., 2007, op. cit.

46. The Human tide: The Real Migration Crisis, op. cit.

47. Cernea M., "Internal Refugee Flows and Development-Induced Population Displacement", Journal of Refugee Studies, vol. 3, n 4, 1990, p. 332 (se référant à Chao L., "State Plans New Rules for Dam Resettlement”, China Daily, n² 2632, 4 janvier 1990).

48. Fruggle R., Smith W.T., Large Dams in Water and Energy Resource Development in the People's Republic of China (PRC), Hydrosult Canada \& Agrodev Canada, 2000 (document national de synthèse préparé pour la Commission mondiale des barrages, Cape Town).

49. Singh Negi N., Ganguly S., Development Projects vs. Internally Displaced Populations in India: a Literature Based Appraisal, COMCAD Working Paper $\mathrm{n}^{\circ} 103$, 2011, p. 6 (www.uni-bielefeld.de/tdrc/ag_comcad/downloads/workingpaper_103_negi_ganguly.pdf, consulté le 21 février 2011).

50. Cernea M., “The Risks and Reconstruction Model for Resettling Displaced Populations”, World Development, vol. 25, n 10, 1997, p. 1570. 
mesures de réinstallation. Toutefois, ces mesures connaissent un échec retentissant parce que les investisseurs sous-estiment souvent les besoins induits par la réinstallation afin de réduire les coûts et présenter une analyse coûtsbénéfices positive.

Les recherches du programme européen EACH-FOR (Environmental Change and Forced Migration Scenarios) sur des cas de réinstallation confirment cette situation ${ }^{51}$. Dans le cas du barrage des Trois-Gorges en Chine, les personnes réinstallées ne pouvaient ni entreprendre une activité agricole pour s'assurer une source fiable de revenus en raison de la mauvaise qualité du sol, ni trouver d'autres opportunités d'emploi. En outre, les familles se sont trouvées séparées et prendre soin des anciens est devenu un problème ${ }^{52}$. De même en Turquie, beaucoup de personnes réinstallées par l'État suite à la construction du barrage Atatürk sont devenues des agriculteurs sans terre ou se sont trouvées sans source alternative de revenus ${ }^{53}$.

Dans les cas de réinstallation en raison de la dégradation environnementale, les résultats ne sont pas nécessairement meilleurs ${ }^{54}$. Le gouvernement chinois a réinstallé, souvent de force, les bergers des zones de pâturage de la Mongolie intérieure dans les zones urbaines en vue d'alléger la pression sur l'environnement et d'améliorer leurs moyens de subsistance. Les éleveurs ont utilisé le programme de réinstallation comme solution à court terme à leurs problèmes économiques mais faute de revenus suffisants et pérennes, ils sont retournés vers leur lieu d'origine dès que les conditions de pâturages se sont améliorées ou ils ont simplement maintenu leurs activités sur leur lieu d'origine en tant que second moyen de subsistance ${ }^{55}$. Les programmes gouvernementaux du Mozambique relocalisant les habitants des zones inondables de la rivière Zambèze ont également connu des résultats très mitigés. Les gens sont retournés à leurs machambas (champs), et ce, malgré le risque d'inondation parce que les conditions du sol et la disponibilité en eau des zones d'accueil étaient très mauvaises ${ }^{56}$. Parfois, ils ont choisi de se rendre quotidiennement sur leurs anciennes parcelles et si cela n'était pas possible en raison de la dis-

51. Stal M., Mozambique Case Study Report, et Dun O., Vietnam Case Study Report, op. cit.

52. Gemenne F., Three-Gorges Dam case study report, Rapport Each-For, 2008.

53. Kadirbeyogulo Z., Turkey Case Study Report, Rapport Each-For, 2008.

54. Pour la présentation d'autres cas, voir Greame H., "Lessons from Past Forced Resettlement for Climate Change Migration”, in Piguet E., Pécoud A., Guchteneire P. (eds.), op. cit., pp. 289-306.

55. Zhang Q., Inner Mongolia Case Study Report, op. cit., p. 22-24.

56. Stal M., Mozambique Case Study Report, op. cit., p. 23-27. Une évaluation pour le district de Mutarara a révélé que dans $62,3 \%$ des cas, le machamba était la principale raison de quitter la nouvelle colonie ; dans $10,4 \%$ des cas, la raison était le tarissement des puits nouvellement créées et dans 9,1\% des cas, la raison était tout simplement l'attachement des personnes à leur terre (Raúl M. Chambote E., Boaventura S., Reassentamento pela Metade no Vale do Zambeze. Um Estudo Independente sobre a Abordagem de Reassentamento Pós-Cheias em Moçambique, à Luz da ERR, 2007/08, Veja Majo, Oxfam, 2008, p. 33).

57. Dans certains cas, les nouvelles colonies se trouvaient à une distance de plus de $30 \mathrm{~km}$ des anciennes maisons, et la moyenne était de 10-15 km (ibid., p. 60). 
tance, ils sont devenus dépendants de l'aide alimentaire ${ }^{57}$. Enfin, les promesses du gouvernement d'engager des projets de développement (par exemple, de nouvelles écoles et centres de santé) n'ont été tenues que très partiellement ${ }^{58}$.

Les projets de réinstallation comme stratégie d'adaptation, même lorsqu'ils sont bien conçus comme dans le cas des Programmes auto-organisés de réinstallation intégrée sur les îles Carteret, échouent souvent parce qu'il n'y a pas assez de terres à disposition ou que le soutien de la population est insuffisant pour y parvenir. Ainsi, les habitants des îles Carteret se sont vus attribuer des terres par l'Église mais elles étaient insuffisantes pour accueillir toutes les familles. Le gouvernement de Papouasie-Nouvelle-Guinée disposait d'un budget pour financer un tel déplacement, par exemple en achetant des terres supplémentaires auprès des communautés de destination au prix du marché. Cependant, ces fonds semblent s'être « évaporés ». Les premières familles réinstallées sont finalement retournées dans leur île qui risque d'être submergée ${ }^{59}$. L'insuffisance des fonds a également été le défi majeur du plan gouvernemental de réinstallation de groupes cibles au Vietnam qui planifiait l'évacuation des habitants des rives érodées du fleuve Mékong. Ces derniers craignaient l'endettement en raison des prêts que ce plan prévoyait d'accorder pour l'achat des terres et la construction des maisons. Souvent pauvres et sans terre, leur principale source de revenu provenait d'emplois journaliers proposés par des voisins en meilleure situation financière. Se déplacer dans la nouvelle zone d'installation leur faisait courir le risque de perdre leurs emplois et leurs réseaux sociaux tout en ayant l'obligation de rembourser leurs prêts forcés. Ainsi, malgré la menace quotidienne des glissements de terrain, beaucoup ont décidé de rester ${ }^{6}$.

Les programmes de réinstallation comme mesures pour lutter contre les effets du changement climatique (autres que les plans de réinstallation préventive dans les zones à risque ou dégradées) ne sont rien d'autre qu'une souscatégorie d'expulsions générées par des projets de développement. En effet, souvent, ces mesures ont un objectif développementaliste comme la construction de barrages, la production d'énergie propre (parcs éoliens, énergie solaire, centrales géothermiques) ou l'investissement agricole à grande échelle dans les agrocarburants. En outre, la reforestation et l'afforestation, qui visent à étendre les puits de carbone ou protéger les bassins versants, les côtes et les berges de rivières, génèrent aussi souvent des déplacements forcés. Toutes ces mesures induisent des changements dans l'utilisation des terres et donc, la plupart du temps, des expulsions.

58. ECHON, Estudo Sobre Abordagem de Intervençãon da Intermon Oxfam no Processo de Reassentamento e Reconstrução Pós Cheias, Maputo, décembre 2008, p. 13.

59. Boege G., Challenges and Pitfalls of Resettlement Measures: Experiences in the Pacific Region, op. cit., p. $32 \mathrm{ff}$.

60. Dun O., Vietnam Case Study Report, op. cit., p. $15 f$. 
La croissance de la production d'agrocarburants, par exemple, a une part considérable dans les tendances actuelles à l'accaparement des terres qui privent les personnes déjà marginalisées de moyens de subsistance essentiels et de ressources naturelles. L'Éthiopie loue au moins 187400 ha de ses terres à différents pays étrangers pour ce nouveau type d'investissement agricole sans tenir compte des moyens de subsistance et de la vulnérabilité des populations nomades et des petits agriculteurs qui vivent sur et de cette terre ${ }^{61}$. Le Kenya est un autre exemple pertinent $62:$ le gouvernement a simultanément mis en œuvre une stratégie de lutte contre le changement climatique et une stratégie de production d'énergies renouvelables avec l'ambition de convertir le pays à une «économie verte ». Dans le delta du Tana, plus de 180000 ha de terres en zone de polyculture sont censés être convertis en plantations de jatropha et de canne à sucre. Le delta est peuplé de petits agriculteurs et d'éleveurs et sert de zone de repli pour les nomades des provinces du Nord pendant les sécheresses. Si les investissements sont réalisés, plus de 1000 familles seront expulsées et plus encore perdront leurs moyens de subsistance. Dans le même temps, l'Éthiopie et le Kenya sont les bénéficiaires récurrents de l'aide alimentaire, comme par exemple pendant la sécheresse sévère qui a touché l'Afrique orientale en 2011.

Le changement d'affectation des terres lié au « verdissement » de l'économie mondiale, qui menace potentiellement les moyens de subsistance de personnes déjà pauvres, ne se limite pas à la réalité quotidienne de l'expansion des agrocarburants. Au Kenya par exemple, des manifestations violentes ont éclaté à Olkaria où 600 familles massaïs ont été expulsées pour permettre l'extension d'une centrale électrique géothermique ${ }^{63}$. Le Kenya veut également atteindre un taux de « couvert arboré » d'au moins $10 \%$ et les plantations de jatropha, qui comptent comme couvert forestier dans le cadre du mécanisme de développement propre en raison de ses effets de captation du carbone, devraient générer des revenus de 46,2 millions de dollars par an ${ }^{64}$. D’autres pays ont également annoncé vouloir accroître considérablement leur couvert forestier : le Togo de 7 à $30 \%$ et la Mauritanie de 3,2 à $9 \%$ d'ici 2050 ; la Chine a l'intention d'augmenter la production forestière de 40 millions d'hectares et son stock forestier de 1,3 milliard de mètres cubes d'ici 2020 ; le Brésil veut doubler les plantations forestières à 11 millions d'hectares en 2020 ; l'Inde

61. GLP, Land grab in Africa: Emerging Land System Drivers in a Teleconnected World, A Joint Research Agenda of IGBP \& IHDP, $\mathrm{n}^{\circ}$ 1, GLP International Project Office and authors, University of Copenhagen, 2010, p. 24.

62. Schade J., Human Rights, Climate Change, and Climate Policies in Kenya: How Climate Variability and Agrofuel Expansion Impact on the Enjoyment of Human Rights in the Tana Delta, Center on Migration, Citizenship and Development, Bielefeld University, décembre 2011, pp. 26-28, 34 and 51.

63. Gitonga A., "KenGen to Resettle 600 Families From Olkaria Plant", The Standard, 23 février 2011 ; Kisero J., "Kenya Must Learn to Interrogate Credentials of Potential Investors”, Daily Nation, 10 août 2011.

64. Schade J., Human Rights, Climate Change, and Climate Policies in Kenya:..., op. cit., p. 27, 34. 
prévoit d'étendre sa couverture forestière d'un tiers ${ }^{65}$. Il est difficile de dire combien de personnes seront touchées mais, comme l'expérience passée le montre, leur nombre risque d'être élevé.

La réinstallation est également un sujet politiquement délicat. Elle peut être utilisée abusivement comme un moyen pour satisfaire des objectifs dissimulés. Par exemple, dans le cas du Mozambique, les personnes réinstallées étaient persuadées que la politique adoptée était une solution d'adaptation au risque accru d'inondations. L'agenda caché semble plutôt répondre à un projet d'évacuation de plaines inondables et fertiles en vue de cultiver la canne à sucre pour la production d'éthanol 66. En d'autres termes, le problème du changement climatique pourrait être abusivement utilisé pour légitimer des plans de réinstallation et répondre à des calculs politiques favorisant un groupe ethnique ou économique en particulier. Dans le passé, la réinstallation a même été utilisée comme instrument stratégique de politique électorale consistant à déplacer des majorités hostiles au pouvoir en place ; ce fut le cas en Somalie sous Siad Barre (président de la République somalienne de 1969 à 1991) ${ }^{67}$. Il n'y a aucune garantie que les mesures de réinstallation justifiées au nom de la politique climatique ne soient pas détournées pour satisfaire des intentions similaires.

Même si les fonctionnaires chargés de mettre en œuvre des plans de réinstallation comme mesure d'adaptation peuvent être sensibles aux questions liées au changement climatique, des considérations à court terme sur les coûts d'options alternatives d'adaptation (comme la création d'un système efficace de gestion de l'eau pour se protéger contre les inondations, la reconstruction et l'amélioration de la protection des endroits endommagés) pourraient les conduire à favoriser le déplacement de populations car à première vue, cette solution semble être la moins coûteuse. Cela pourrait devenir une réalité, en particulier en ce qui concerne les groupes socialement vulnérables qui n'ont pas la possibilité de se faire entendre pour exiger des solutions in situ ou de plans de réinstallation susceptibles d'améliorer véritablement leurs conditions de vie. Comme le montre l'expérience des habitants afro-américains des bords de la rivière Mississippi à la Nouvelle-Orléans, le problème ne se pose pas seulement aux pauvres dans les pays en voie de développement ${ }^{68}$. Alors que leurs

65. L'information est basée sur McDowell, Climate Change Adaptation and Mitigation:..., op. cit., 8f. Son examen des programmes d'action nationaux d'adaptation (PANA), des mesures d'atténuation nationales appropriées (MAMA) et des documents stratégiques sur le changement climatique des pays à forte industrialisation (Brésil, Afrique du Sud, Inde et Chine) révèle qu'au moins 22 pays envisagent de grands projets pouvant impliquer des expulsions et des réinstallations. Cependant, les détails sur leur mise en ouvre sont rarement fournis.

66. Raúl M. Chambote E., Boaventura S., Reassentamento pela Metade no Vale do Zambeze..., op. cit., p. 11. Pendant la période coloniale, la région avait déjà été utilisée pour la culture de la canne à sucre.

67. Nannini Vance J., Decisions in Operations Other Than War: The United States Intervention in Somalia, Storming Media, Pentagon Reports, n A966482, 1994.

68. Elliot J. R., Pais J., "Race, Class, and Hurricane Katrina: Social Differences in Human 
quartiers n'ont pas été reconstruits après l'ouragan Katrina en 2005, ceux de leurs voisins de la classe moyenne ont fait l'objet d'une protection renforcée. Cela a finalement contribué à une stratification urbaine.

Pour résumer, les mesures de réinstallation sont souvent mal appliquées tant du point de vue des procédures suivies que des résultats obtenus. Les gens ne sont ni consultés ni informés, ils ne reçoivent pas de logement adéquat ni de moyens de subsistance suffisants sur leur nouveau lieu d'installation. Pendant l'évacuation, ils peuvent même être victimes de violence et de corruption par les institutions responsables de sa mise en ouvre. S’ils se retrouvent loin de leur lieu d'origine, ils se voient souvent privés de biens essentiels à leur subsistance et/ou d'infrastructures sociales comme les écoles et les hôpitaux. Et même s'ils peuvent être soulagés des pressions climatiques, ils sont susceptibles de devenir plus vulnérables à d'autres éléments comme, par exemple, le manque d'eau. En outre, le risque d'émergence de conflits et la dégradation de l'environnement pourraient croître dans les communautés d'accueil. Selon Michael Cernea, les risques liés à la réinstallation sont nombreux : privation de terres, chômage, itinérance, augmentation de la morbidité et de la mortalité, insécurité alimentaire, perte d'accès à la propriété commune et désagrégation sociale, le degré de gravité variant selon les groupes vulnérables (femmes, enfants, populations autochtones, etc.) ${ }^{69}$.

Eu égard à ces constatations, il semble que renforcer les mesures de réinstallation dans le cadre de la CCNUCC constitue une opération à double tranchant. Du point de vue des groupes vulnérables, nous pouvons qualifier une telle répercussion négative des politiques climatiques d'impact de sixième ordre du changement climatique.

\section{Comment placer les personnes vulnérables au centre du débat : l'apport de Sen}

Il apparaît comme une ironie du sort que les sujets directement concernés par le débat sur les migrants climatiques, ceux qui sont particulièrement vulnérables aux impacts du changement climatique, risquent de surcroît d'être victimes des politiques. Souvent, les groupes vulnérables vivent dans des zones côtières périphériques des grandes villes qui sont sujettes à divers dangers comme l'érosion. Or les interventions visant à améliorer la protection du littoral sont susceptibles de concerner avant tout les sites industriels et les éta-

Responses to Disasters", Social Science Research, vol. 35, n² 2, 2006, pp. 295-321.

69. Cernea M., "The Risks and Reconstruction Model for Resettling Displaced Populations”, op. cit., pp. 1572-1576. Pour en savoir plus sur les défis et les pièges de la réinstallation, voir Cernea, M., McDowell C. (eds.), Risks and Reconstruction: Experiences of Resettlers and Refugees, Washington D.C, Banque mondiale, 2002 ; Cernea M., Kanbur R., An Exchange on the Compensation Principle in Resettlement, Department of Applied Economics (Cornell University), Working Paper 202-33, 2002 ; McDowell C., Morell G., Displacement Beyond Conflict: Challenges for the 21st Century, Oxford/New York, Berghan Books, 2010. 
blissements de l'arrière pays. Ce constat vaut pour la protection des berges de rivières densément peuplées. Les zones rurales les plus vulnérables au changement climatique comptent souvent des petits exploitants et petits éleveurs sans titres légaux de propriété. Dans de nombreux cas, c'est parce qu'ils ont déjà été repoussés vers des terres moins fertiles qu'ils sont maintenant susceptibles de souffrir de l'aggravation de l'aridité. Les techniques de stockage de l'eau pour assurer l'irrigation des cultures tendent à bénéficier à l'agriculture à grande échelle, existante ou en projet (comme les cultures de jatropha et les plantations de cannes à sucre) plutôt qu'aux petits agriculteurs. Les gens défavorisées et les paysans «non qualifiés » trouvent rarement un travail décent et un emploi fiable sur les nouvelles plantations.

Dans ce contexte, il semble opportun de rappeler quelques idées déjà exposées lors de la discussion critique de la perspective malthusienne sur le problème de la famine. Dans les années 1970, l'insécurité alimentaire était selon la définition que Malthus donne du problème - principalement gérée comme une question de productivité agricole et de disponibilité alimentaire par habitant (population/ratio alimentaire). Selon le discours $P \& E$, le décalage entre la disponibilité en ressources et la population pourrait être abordé sous l'angle des solutions techniques. Ainsi, les réponses aux famines, appuyées par les politiques de la Banque mondiale, ont fait la promotion des techniques de "révolution verte ", la privatisation des droits fonciers, la planification familiale et l'importation subventionnée d'aliments ${ }^{70}$. Cette approche a été rapidement contestée. À travers son ouvrage sur les famines, Amartya Sen est celui qui a formulé les critiques les plus abouties. Il a montré que (a) de nombreuses famines sont dues à des facteurs tels que les crises politiques, la récession économique, l'inflation ou la guerre et non seulement aux sécheresses et aux catastrophes naturelles; (b) les famines n'affectent pas la population d'un pays de manière égale car ses segments présentent différents degrés de vulnérabilité sociale selon les «droits » qu'ils peuvent faire valoir ; (c) ces droits sont fortement réglementés et délimités par les institutions et par un accès (inégal) à ces mêmes droits ${ }^{71}$. Par conséquent, l'augmentation de la production agricole ne

70. Hartmann B., "Rethinking the Role of Population in Human Security", op. cit., p. 196f. Pour plus d'éléments : Journal of Agricultural Economics, vol. 27, n 1, 1976 (en particulier la contribution de McInerney J., "The simple analytics of natural resource economics" (pp. 3152), de Sinha R.P., "World food security" et de Norse D., "Development strategies and the world food problem" (pp. 137-158). Un exemple de document politique représentant cette ligne de pensée est celui de la FAO, Plan of Action on the World Food Security, resolution 3/79 of the 20th session of the committee on world food security, Rome, FAO, 1979.

71. Sen A., Poverty and Famines. An Essay on Entitlement and Deprivation, Oxford, Clarendon Press, 1981 ; Sen A., Food, Economics and Entitlements, UNU World Institute for Development Economics, WIDER Working Papers, WP $n^{\circ} 1,1986$. Le dernier point, sur les institutions, recoupe en partie la critique de l'approche de l'écologie politique au sein des études P\&E et de ses fondements théoriques de la dépendance, qui mettent l'accent sur les inégalités structurelles comme cause principale de la pauvreté et de la dégradation de l'environnement (Sherbinin A., Carr D., Cassels S., Jiang L., "Population and Environment", op. cit., p. 349, faisant référence à Carole J., "Fourth Theories of Population Change and the Environment", Population and Environment, vol. 16, n 1, 1994, pp. 61-90). 
peut suffire à améliorer la situation de ceux qui souffrent de la faim. Pour révéler les vraies raisons pour lesquelles les pauvres ne peuvent acquérir suffisamment de nourriture, une analyse de la cartographie de leurs droits est nécessaire. Il est clair que les relations socio-culturelles et les pratiques discriminatoires des institutions (par exemple l'accès à l'éducation, au marché du travail, au capital ou aux institutions politiques) jouent un rôle majeur.

Ses travaux et ceux de ses disciples ont ainsi déplacé l'attention du niveau macro au niveau micro, ouvrant ainsi la voie à une perspective différente sur les causes de la faim. Cela a considérablement influencé le discours politique sur la sécurité alimentaire et l'approche micro a même permis la reconnaissance du droit à l'alimentation comme un droit de l'homme fondamental 72. Le facteur humain est ainsi placé au centre de l'attention en trouvant son expression savante, entre autres, dans l'approche des moyens d'existence durables (sustainable livelihood approach), l'approche des capabilités de Sen et dans les approches du développement fondées sur les droits. En effet, les plus éminents chercheurs en matière de vulnérabilité sociale au changement climatique et les experts en matière de réinstallation suivent cette ligne de pensée. En ce sens, Neil Adger et Michael Kelly ont écrit que « la réponse au changement climatique est facilitée ou limitée par la même architecture des droits qui influence l'adaptation à d'autres contraintes sociales et environnementales ${ }^{73}$ ».

Aujourd'hui, le régime de la CCNUCC prévoit une série de mécanismes pour gérer les émissions de GES et préserver au mieux l'atmosphère mondiale et l'humanité. Bien que les groupes vulnérables bénéficient indirectement et sur le long terme de ces efforts, ceux-ci sont encore moins tangibles que le ratio alimentaire à l'échelle nationale ou mondiale. Il nous semble nécessaire aujourd'hui d'examiner comment les politiques et instruments de lutte contre le changement climatique (par exemple le Fonds vert pour le climat, le Fonds

72. En 1983, la FAO et le Conseil mondial de l'alimentation ont recommandé de redéfinir la notion de "sécurité alimentaire » et au milieu des années 1980, une nouvelle définition a également été adoptée par la Banque mondiale. En 1996, durant le Sommet mondial de l'alimentation et, en 2007, par la FAO, la définition a été de nouveau modifiée et comprend aujourd'hui la disponibilité en nourriture, l'accès physique et économique à la nourriture, les besoins alimentaires et les préférences culturelles. Ces éléments reflètent le contenu du droit à l'alimentation tel qu'il a été défini par l'Observation générale n ${ }^{\circ} 12$ de 2002 du Comité pour les droits économiques, sociaux et culturels (CESCR) et par des universitaires engagés. FAO, Approaches to World Food Security, Economic and Social Development, Paper n ${ }^{\circ} 32$, Rome, 1983 ; FAO, Déclaration de Rome sur la sécurité alimentaire mondiale, WSF 96/3, Rome, 1996 ; CESCR, The Right to Adequate Food, General Comment n 12, E/C.12/1999/5, 1999. Pour l'évolution de la notion de sécurité alimentaire, voir Maxwell S., "Food Security: A Post-Modern Perspective”, Food Policy, vol. 21, n² 2, 1996, p. $156 f$.

73. Adger W. N., Kelly P. M., "Social Vulnerability to Climate Change and the Architecture of Entitlements", Mitigation and Adaptation Strategies for Global Change, vol. 4, n 3-4, 1999, pp. 253-266, 255. Parmi les chercheurs qui travaillent sur la vulnérabilité sociale et qui sont influencés par l'approche de Sen, nous pouvons citer : Bohle H. G., Downing T. E., Watts M. J., "Climate Change and Social Vulnerability: Toward a Sociology and Geography of Food Insecurity”, Global Environmental Change, vol. 4, nº 1, 1994, pp. 37-48; Wisner B., Blaikie P. M., Cannon T., Davis I., At Risk: Natural Hazards, People's Vulnerability and Disasters, Londres, Routledge, 2e édition, 2006. 
d'adaptation, mécanisme de développement propre (MDP), programme de réduction des émissions liées au déboisement et à la dégradation des forêts (REDD +), etc.) refaçonnent l'architecture des droits et interagissent avec les contraintes existantes et les opportunités des segments les plus vulnérables de la population. C'est une question de cohérence politique et de respect des droits de l'Homme de faire en sorte que les politiques climatiques n'altèrent pas la capacité de résilience des groupes vulnérables. Pour y parvenir, il est nécessaire de mettre à nouveau l'accent sur le niveau micro. Et, comme les discussions sur la sécurité alimentaire nous l'enseignent, les droits de l'Homme peuvent servir de guide utile pour corréler les approches globales aux défis que rencontrent les gens au niveau local.

\section{Protéger le migrant des politiques climatiques}

Les droits de l'Homme, en tant que cadre normatif, s'intègrent très bien au cadre analytique d'approches comme celle des moyens d'existence durables en raison de leur focalisation sur l'individu et le niveau micro. Entre le régime des droits de l'Homme et le régime climatique, l'échange a été jusqu'ici limité. Les rapports du GIEC et de la CCNUCC ne se réfèrent que de manière sporadique et parcimonieuse aux droits humains. Néanmoins, depuis la sortie de l'AR4 du GIEC en septembre 2007, qui a déclaré que les mesures d'atténuation devaient se traduire par des résultats socialement acceptables, les efforts consistant à prendre en considération la relation entre changement climatique et droits de l'Homme sont notables ${ }^{74}$. En novembre 2007, les petits États insulaires ont demandé au Haut-Commissariat des Nations Unies aux droits de l'homme (HCDH) de produire un rapport sur les atteintes aux droits de l'Homme générées par le changement climatique, initiative suivie par le Conseil de droits de l'Homme des Nations Unies (CDH) en mars 2008. Le rapport du $\mathrm{HCDH}$ a été publié en janvier 2009, suivi d'une résolution du $\mathrm{CDH}$ sur le changement climatique et les droits de l'Homme ${ }^{75}$. En parallèle, les publications scientifiques se sont multipliées ${ }^{76}$. Le rapport du $\mathrm{HCDH}$ s'attache surtout aux principaux droits de l'Homme menacés par le changement climatique, mais il souligne également le fait que les mesures d'interven-

74. ICHRP, Climate Change and Human Rights: A Rough Guide, Versoix, 2008, p. $29 f$ (se référant au IPCC AR4, WGIII, p. 696).

75. Hunter D., "Human rights implications for climate change negotiations", op. cit., p. 337f.; HRC, Resolution 10/4. Human rights and climate change, A/HRC/RES/10/4, mars 2009. Il convient de noter que le CERD a souligné les effets négatifs des plantations de palmiers à huile sur les droits des autochtones en août 2007, peu avant que le AR4 du GIEC soit publié. Ce fut cependant sans référence aux politiques de lutte contre le changement climatique (CERD, Concluding observations of the Committee on the Elimination of Racial Discrimination: Indonesia, CERD/C/IDN/CO/3, août 2007, 3f.).

76. ICHRP, Climate Change and Human Rights..., op. cit. ; Hunter D., "Human rights implications for climate change negotiations", op. cit. ; Loftus-Farren Z., McKiernan C., Protecting People and the Planet: A Proposal to Address the Human Rights Impacts of Climate Change Policies, International Human Rights Law Clinic/Miller Institute for Global Challenges and the Law/Center for Law \& Global Justice, décembre 2009 ; Humphreys S. (ed.) Human Rights and Climate Change, Cambridge, Cambridge University Press, 2010. 
tion elles-mêmes peuvent potentiellement mettre en péril les droits des personnes vulnérables et des populations autochtones 77 .

Ces publications ont eu une certaine influence et certains progrès ont été réalisés lors de la COP-16 à Cancun 2010 78. Pour la première fois, le texte de l'AWG-LCA mentionne explicitement la « migration climatique » et évoque également la question des droits de l'homme ${ }^{79}$, en référence à la conception des projets d'adaptation et des projets REDD (Reducing emissions from deforestation and forest degradation). Plus particulièrement, pour les projets REDD, des «garanties » relatives aux droits des peuples autochtones ont été demandées 80 . Malheureusement, les termes utilisés dans le document final de l'AWG-LCA ne correspondent pas au vocabulaire codifié des droits de l'Homme (par exemple, la formule « la nécessité de respecter " a été préférée plutôt que « les obligations des États de respecter, protéger et appliquer les droits de l'homme »). Pour les mesures d'atténuation, seule est mentionnée la nécessité de prendre en considération les informations transmises par les populations affectées ${ }^{81}$.

Conscients des lacunes en matière de protection des personnes déplacées au sein du régime climatique, les experts en matière de réinstallation ont récemment commencé à préconiser des approches alternatives développées au cours des années 1990 82. Celles-ci promeuvent la « justice sociale et les normes d'équité et de respect pour les droits civiques et les droits des gens » en vue de résoudre la " pathologie du développement » et éviter que «certaines personnes profitent des gains du développement, tandis que d'autres en supportent les coûts 83 ». Ces approches peuvent être appliquées à tout type de réinstallation, y compris dans le cadre de la politique climatique. Les experts recommandent entre autres (1) que les groupes cibles et les communautés d'accueil participent à la prise de décisions concernant la façon de pro-

77. HRC, Report of the Office of the High Commissioner for Human Rights on the Relationship between Climate Change and Human Rights, A/HRC/10/61, 15 janvier 2009, p. 8-15, 22.

78. Pour une analyse détaillée, voir CIEL, Analysis of Human Rights Language in the Cancun Agreements (COP-16), Center for Environmental Law, février 2011.

79. UNFCCC, The Cancun Agreements: Outcome of the work of the Ad Hoc Working Group on Long-term Cooperative Action under the Convention (AWG-LCA), Decision 1/CP.16, adopté à la Conférence des Parties sur sa seizième session, tenue à Cancún du 29 novembre au 10 décembre 2010, $\mathbb{~} .7,8,12$.

80. Ibid., annexe I, $\$ 2(\mathrm{c})$ et (d). Les directives concernant les garanties REDD attendues pour le COP-17 n'ont pas encore été livrées (ibid., \$.71(d)). REDD Monitor, REDD New From The Conference of Polluters (Durban COP-17), 8 décembre 2011 (www.reddmonitor.org/2011/12/08/redd-news-from-the-conference-of-polluters-durban-cop-17-8december-2011/, consulté le 21 février 2011).

81. Ibid., $\$ .92$ et annexe III, $\mathbb{S} .1(\mathrm{~h})$.

82. Sherbinin et al., op. cit.

83. Cernea M., "Risks, Safeguards, and Reconstruction: A Model for Population Displacement and Resettlement”, in Cernea M., McDowell C. (eds.), Risks and Reconstruction: Experiences of Resettlers and Refugees, op. cit., p. 12 ; Cernea M., "The Risks and Reconstruction Model for Resettling Displaced Populations”, op. cit., p. 1569. Voir aussi McDowell C. (ed.), Understanding Impoverishment - The Consequences of Development-Induced Displacement, New York, Berghahn Books, 1996. 
céder et le choix du lieux de réinstallation ; (2) que des compensations et des programmes de développement accompagnant la réinstallation soient élaborés pour assurer et améliorer les moyens de subsistance et qu'ils soient intégrés aux politiques mises en œuvre par ailleurs (par exemple dans les politiques de protection du littoral, de reforestation ou d'afforestation, les projets MDP ou les projets de stockage de l'eau pour l'irrigation, etc.) ; (3) que des études d'impact environnemental et social (EIES) soient menées pour permettre d'évaluer les initiatives de réinstallation ; et (4) qu'une formation interdisciplinaire soit offerte aux professionnels de la réinstallation et les autorités responsables. Les principes directeurs relatifs au déplacement de personnes à l'intérieur de leur propre pays fondés sur les droits de l'Homme fournissent un cadre supplémentaire, en particulier pour gérer les populations déplacées au cours d'une procédure d'expulsion et dans des camps d'évacuation suite à une catastrophe.

Dans un effort de recherche de lignes directrices plus appropriées, il nous semble par ailleurs nécessaire de renforcer les références directes aux droits de l'Homme et de prendre en considération les principes de base et les directives sur les expulsions forcées proposées par l'ancien rapporteur spécial des Nations Unies sur le droit au logement convenable, Miloon Kothari ${ }^{84}$ (20002008). La prise en compte des droits de l'Homme dans les cas de réinstallations liés aux politiques climatiques présenterait, entre autres, les avantages suivants.

Tout d'abord, une EIES dont les critères d'évaluation seraient fondés sur les droits de l'Homme permettrait de rehausser les normes nationales et les conditions de vie des gens là où elles sont peu considérées. Par ailleurs, les droits de l'Homme mettent l'accent sur le principe de non-régression et permettent d'assigner des responsabilités et des obligations, même en l'absence de structures claires de responsabilité ${ }^{85}$. En outre, le respect de la vie des gens n'est plus un acte généreux de protection sociale ou un service social offert par les investisseurs, mais un devoir envers les titulaires de droits. Ces derniers pourraient entamer des poursuites judiciaires s'ils estiment que leurs droits ont été violés ou que leur protection n’a pas été assurée.

Deuxièmement, la prévention doit être renforcée pour éviter un déplacement inutile ou une réinstallation pour de «fausses » raisons. Les directives de Kothari rappellent que les autorités ont l'obligation de prouver que la réinstallation ne peut être évitée et évoquent le droit des personnes concernées à proposer des alternatives au déplacement (ce qui implique que leur participation soit garantie). Les directives sont en effet très détaillées concernant les prin-

84. HRC, Implementation of General Assembly, Resolution 60/251 of 15 March 2006, Report of the Special Rapporteur on Adequate Housing as a Component of the Right to an Adequate Standard of Living, Miloon Kothari, A/HRC/4/18, 5 février 2007.

85. Walker S., The Future of Human Rights Impact Assessments of Trade Agreements, School of Human Rights, Research Series, vol. 35, Utre, 2009, pp. 45-47. 
cipes d'information et de participation, ainsi que sur le calendrier et la nécessité d'un consentement préalable libre et éclairé ${ }^{86}$.

Troisièmement, les directives de Kothari insistent sur le droit à la sécurité de la propriété. Il s'agit d'un point très important parce que la terre est au cœur du problème. Les mesures d'adaptation et d'atténuation comportent un risque élevé d'expulsion parce qu'elles impliquent l'utilisation de territoires très étendus, qui prive les gens de leurs terres ou des ressources dont ils dépendent. Le non-respect généralisé de la garantie de la propriété et des droits d'accès coutumiers sur les ressources naturelles est souvent le contexte au sein duquel les expulsions et la réinstallation deviennent la solution « facile ». En raison de la faiblesse, voire de l'absence de régimes fonciers (en Afrique notamment), on peut d'ailleurs se demander si les avantages potentiels des mécanismes de Kyoto bénéficieraient aux communautés ou aux seuls gouvernements et investisseurs privés ${ }^{87}$.

Quatrièmement, dans le cas où la réinstallation se révèle être la meilleure solution, des directives détaillées sont élaborées tant en ce qui concerne le processus de préparation de la réinstallation que le processus de départ (sur la base des principes directeurs relatifs aux déplacements internes) et les exigences du nouvel emplacement. Il faut aussi garantir le respect des droits fondamentaux tels que le droit à l'alimentation, à l'eau, au logement et à la santé. Ces directives rappellent par ailleurs la nécessité de considérer le calendrier des populations concernées (les récoltes, les examens, les élections, etc.), la qualité de l'environnement du lieu de destination, la distance qui le sépare du précédent lieu de vie (qui doit être la plus courte possible), les alternatives en termes de revenus et de mobilité ainsi que tout un ensemble d'autres considérations permettant d'améliorer le facteur humain ${ }^{88}$.

Bon nombre de ces points sont également inclus dans les approches des experts en réinstallation et sont désormais incontournables dans la planification du développement progressif, sans pour autant être nécessairement considérés comme des droits. En effet, il n'est pas facile d'appliquer ces directives de manière efficace parce qu'elles n'ont aucun caractère obligatoire. Il en va de même des droits de l'Homme. La plupart des États sont signataires de la Déclaration universelle des droits de l'homme (1948), du Pacte international relatif aux droits civils et politiques ainsi que du Pacte international relatif aux droits économiques, sociaux et culturels (1966) et doivent donc s'y conformer. Les droits de l'Homme convertissent les normes fondées sur ces droits en obligation. Si les litiges sont rejetés au niveau national, les titulaires des droits peuvent s'adresser aux tribunaux supérieurs des droits de l'Homme, du moins

86. HRC, Implementation of General Assembly, op. cit.

87. Willy L. A., "Custom and Commonage in Africa: Rethinking the Orthodoxies", Land Use Policy, vol. 25, n 1, 2008, pp.43-52.

88. Ibid., p.33. 
en Europe, en Afrique et en Amérique. Certes, la Cour interaméricaine des droits de l'homme a rejeté l'affaire des Inuits accusant les États-Unis de ne pas avoir évité les impacts du changement climatique sur l'Arctique ${ }^{89}$. L'une des raisons était qu'il n'est pas possible d'attribuer les impacts climatiques locaux aux émissions d'un État particulier. Mais pour ce qui concerne les expulsions ou d'autres impacts locaux liés aux politiques climatiques, le problème ne se pose pas et les juridictions régionales et nationales peuvent effectivement se prononcer 90 .

Les droits de l'Homme peuvent aussi servir de guide au niveau international. D’une part, Biermann et Boas ont raison de souligner que le déplacement dans les pays en développement est fortement lié à des problèmes de corruption, de mauvaise gouvernance et d'absence d'État de droit. D'autre part, les pays signataires de l'Annexe 1 de la CCUNCC ne peuvent pas se dégager de leur responsabilité :

(1) les mesures d'atténuation sont bénéfiques pour l'atmosphère terrestre dans son ensemble, c'est-à-dire aussi pour les pays développés, et les systèmes d'échange de quotas d'émission profitent à leurs entreprises par leur implication dans des projets d'atténuation. En effet, ce sont ces pays qui bénéficient le plus des mesures d'atténuation appliquées dans les pays non inclus dans l'Annexe 1 dans la mesure où les efforts d'adaptation de leurs économies et de leurs modes de consommation seront moindres pour atteindre les objectifs d'émissions. Le soutien proposé aux pays tiers devrait aussi répondre aux exigences en termes de droits de l'Homme.

(2) En vertu du principe de « responsabilité commune mais différenciée » de la CCNUCC, les pays développés sont tenus d'aider les pays en développement dans leurs efforts d'adaptation. Dans le cadre du régime des droits de l’Homme, les pays développés doivent être obligés de soutenir la réalisation progressive des droits de l'homme.

(3) Récemment, la Commission internationale des juristes a publié des principes sur les obligations extraterritoriales des États (OTE) ${ }^{91}$. Ces obliga-

89. Hunter D., "Human rights implications for climate change negotiations", op. cit., p. 336, 343.

90. Hunter donne l'exemple de la revendication examinée par la Haute Cour fédérale du Nigeria sur le gaz naturel libéré lors de l'extraction du pétrole (ibid., p. 331). Un autre exemple est la décision de la Commission africaine des droits de l'homme en faveur de la Communauté Edorois et contre le gouvernement du Kenya qui l'avait expulsée pour établir une réserve naturelle. Les Edorois ont pu ainsi revenir sur leur terre et ont dû être dédommagés pour l'expulsion (Human Rights Watch, "Kenya: Landmark Ruling on Indigenous Land Rights. African Human Rights Commission Condemns Expulsion of Endorois People for Tourism", 4 février 2010 (www.hrw.org/news/2010/02/04/kenya-landmark-ruling-indigenous-landrights, consulté le 21 février 2011).

91. ICJ, Maastricht Principles on Extraterritorial Obligations of States in the Area of Economic, Social and Cultural Rights, International Commission of Jurists, septembre 2011 (http://www.rtfn-watch.org/uploads/media/Maastricht_ETO_Principles_EN.pdf, consulté le 21 février 2011) 
tions sont en attente d'une reconnaissance plus large, afin d'aider à renforcer la responsabilité transnationale dans un monde globalisé. Elles obligent les États à prévenir les violations des droits de l'homme dans de pays tiers par des acteurs relevant de leur juridiction et à veiller à ce que les accords internationaux prennent en compte les droits de l'homme et ne leur portent pas préjudice.

En somme, il n'y a aucune raison que les groupes les plus vulnérables subissent les coûts sociaux des mesures d'atténuation et d'adaptation pour que nous puissions exercer nos droits d'émission.

\section{Conclusion}

Il est crucial que toute solution politique envisagée intègre la question des droits de l'Homme. Le principe « ne pas nuire » basé sur les droits de l'Homme devrait guider des politiques garantissant que les personnes concernées ne soient pas privées de leurs droits sans une compensation adéquate. C'est une question de cohérence politique et de respect des droits de l'Homme que de prendre en compte les impacts négatifs des politiques climatiques sur les migrations forcées et les droits des populations locales. Par ailleurs, ce type d'analyse doit également être appliqué aux propositions avancées dans le débat politique sur les migrants climatiques, en particulier en ce qui concerne la réinstallation. Malgré les bonnes intentions, le risque que les politiques publiques soient mises en œuvre au détriment des personnes qu'elles sont censées soutenir doit être évalué. Faute de quoi elles pourraient aboutir à l'accroissement de la vulnérabilité plutôt qu'à l'amélioration des conditions de vie des populations et à la résilience des communautés. 